\title{
Editorial
}

Monatsschr Kinderheilkd 2020 • 168:826-827 https://doi.org/10.1007/s00112-020-00867-2 Online publiziert: 6. Februar 2020

(c) Springer Medizin Verlag GmbH, ein Teil von Springer Nature 2020

J. Dötsch

Klinik und Poliklinik für Kinder- und Jugendmedizin, Uniklinik Köln, Köln, Deutschland

\section{Unterschied zwischen Ökonomisierung im und Kommerzialisierung des Gesundheitswesens}

Widerspricht die ärztliche Ethik der Ökonomisierung im Gesundheitswesen? Diese Frage würden die meisten von uns auf Anhieb wohl mit Ja beantworten. Ohne Zweifel beeinflusst Ökonomie ärztliches Handeln auch in unserem Land.

Grundsätzlich ökonomisiert jeder Einzelne von uns seine Situation im medizinischen Kontext, unabhängig vom Kostendruck im Gesundheitssystem. Beispiele hierfür sind Gehaltsverhandlungen oder die Optimierung der Erlössituation bei selbstständiger ärztlicher Tätigkeit. Insofern wirkt die Diskussion um den unüberbrückbaren Gegensatz von Ethik und Ökonomie bisweilen abgehoben.

Um dieser Problematik zu entgehen, differenziert Franz Staudt in seiner Arbeit „Ökonomie in der Medizin ethische Aspekte“ ganz klar zwischen Ökonomisierung im Gesundheitswesen und Kommerzialisierung des Gesundheitswesens. Ökonomisierung, wenn sie zum Wohle des Patienten eingesetzt wird, kann durchaus wertvoll und richtig sein kann, z. B. um durch sinnvolle Planung von diagnostischen und therapeutischen Maßnahmen Übertherapien $\mathrm{zu}$ vermeiden. Dem steht Kommerzialisierung gegenüber, insbesondere wenn gewinnorientierte Finanzinvestoren bzw. Konzerne, einer schleichenden Industrialisierung des Gesundheitswesens den Weg bereiten und auf Basis von Renditeerwartungen Reinvestitionen ins Gesundheitssystem vermindern oder verhindern.
Die Besonderheit des Beitrags macht sicherlich aus, dass Staudt Konzepte zur Vermeidung von Übertherapie beschreibt, wie das "Choose-wisely“Programm, bei dem die kluge Entscheidungsfindung gezielt Diagnostik und Therapie unterlässt, die keine tragende Rolle im Behandlungsprozess des Patienten spielen.

Am Beispiel der Entwicklung von Kinder- und Jugendmedizin im Zeitalter der Ökonomisierung, mehr aber noch der Kommerzialisierung illustriert der Autor die Gefahren und Risiken für Versorgungsstrukturen in vermeintlich unlukrativen Bereichen. Der Widerspruch, dass unser Gesundheitssystem gerade die Altersgruppen stiefmütterlich behandelt, die durch gesundheitserhaltende und präventive Maßnahmen langfristig am meisten profitieren, wird in dem Betrag eindrücklich unterstrichen.

\section{》) Die Zeitlosigkeit ethischer Prinzipien hat auch für den Gesundheitssektor zu gelten}

Können die Probleme der Kommerzialisierung im Gesundheitswesen an übergeordneter Stelle überhaupt gelöst werden? Sicherlich nur schwer oder gar nicht, wo die Kommerzialisierung einzelner Bereiche (insbesondere der Bereich von Medizinprodukten wird hier immer wieder genannt) der Stabilisierung eines Klinikums in der Gesamtheit dienen soll. Ist Letzteres vielleicht sogar eine „gute“ oder „unvermeidbare“ Kommer- 


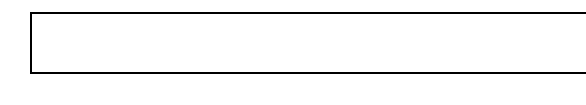

zialisierung, um Schaden vom Großen und Ganzen abzuwenden und damit das ethische Dilemma in unterfinanzierten Bereichen des Gesundheitssystems z. T. von den Schultern der Behandler zu nehmen? Hierauf können die aktuelle Diskussion und auch die Publikation von Staudt naturgemäß keine nachhaltige Antwort finden, bleiben doch die bestehenden Grundsätze der Ethik unverändert.

Die Zeitlosigkeit ethischer Prinzipien, wie sie auch für den Gesundheitssektor gültig sein sollten, unterstreicht Staudt mit dem kategorischen Imperativ, der allerdings in einer Zeit formuliert wurde, in der die Mechanismen der heutigen Gesellschaftsstruktur und des heutigen Gesundheitssystems auch nicht in Ansätzen zu erkennen waren.

Insofern bleibt für den heute handelnden Arzt das Dilemma bestehen, ein grundsätzlich ökonomisch Handelnder $\mathrm{zu}$ sein und dies mit dem eigenen $\mathrm{Ge}$ wissen vereinbaren zu müssen. Trotz der (auch nicht zu erwartenden) vollständigen Unterscheidung zwischen Ökonomisierung und Kommerzialisierung des Gesundheitssystems bietet die Differenzierung, die Staudt vorschlägt, in meinen Augen eine zielführende Möglichkeit für den modernen Arzt, seine Rolle in ethisch vertretbarem Rahmen zu definieren.

\section{Korrespondenzadresse}

\section{Prof. Dr. J. Dötsch}

Klinik und Poliklinik für Kinder- und

Jugendmedizin, Uniklinik Köln Kerpener Str. 62, 50937 Köln, Deutschland joerg.doetsch@uk-koeln.de

Interessenkonflikt. J. Dötsch ist als Direktor einer Klinik für Kinder- und Jugendmedizin dem gesetzlichen Gebot zur Wirtschaftlichkeit verpflichtet.

\section{In eigener Sache}

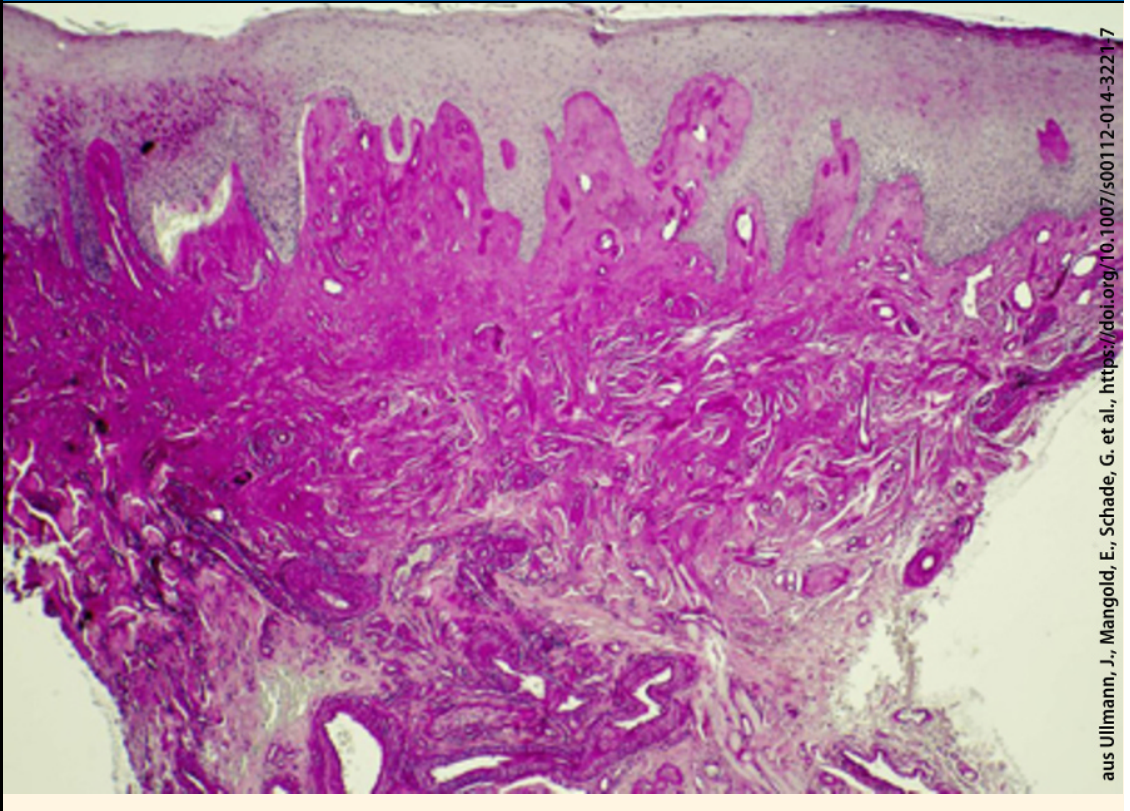

\section{Ihr Fall in Monatsschrift Kinderheilkunde - reichen Sie Ihr Manuskript für „Bild und Fall" ein}

Sie hatten einen interessanten Fall in Ihrer Praxis? Einen ungewöhnlichen Krankheitsund Behandlungsverlauf? Instruktives Bildmaterial, an dem sich das diagnostische und therapeutische Vorgehen darstellen lässt?

Bereiten Sie Ihr Fallbeispiel für die Rubrik „Bild und Fall“ auf und lassen Sie mehr als 18.000 Leserinnen und Leser der Monatsschrift Kinderheilkunde an Ihren Erfahrungen teilhaben!

Der Beitrag gliedert sich in zwei Hauptteile:

- Im ersten Teil wird der Fall kurze dargestellt mit Anamnese, klinischem Befund und Diagnostik. Der Leser soll durch Fallbeschreibung und Bildmaterial alle nötigen Informationen erhalten, um selbst eine Diagnose zu stellen.

- Erst im zweiten Teil wird die Diagnose genannt. Therapie und Verlauf des vorgestellten Falls werden beschrieben; der Leser erhält weitere Hintergrundinformationen wie z.B. Pathogenese, Differentialdiagnose und weiterführende Diagnostik.

Einen detaillierten Leitfaden finden Sie auf www.MonatsschriftKinderheilkunde.de

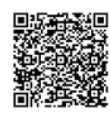

Die Manuskripte werden von 2 unabhängigen Gutachtern geprüft. Sie erhalten detaillierte Rückmeldungen und konstruktive Anmerkungen zur Verbesserung Ihres Beitrags.

Reichen Sie Ihren Beitrag online ein: https://www.editorialmanager.com/moki/

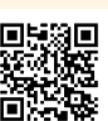

Fragen zur Rubrik beantwortet die Redaktion: Christine Braun

E-Mail: christine.braun@springer.com 